\title{
Metabolic Reduction in the Posterior Cingulate Cortex in Very Early Alzheimer's Disease
}

Satoshi Minoshima, MD, PhD, ${ }^{*}$ Bruno Giordani, $\mathrm{PhD}, \dagger$ Stanley Berent, PhD, $\dagger \neq$ Kirk A. Frey, MD, PhD, ${ }^{*} \ddagger$

Norman L. Foster, MD, $\ddagger$ and David E. Kuhl, MD*

This study investigated cerebral glucose metabolism in very early Alzheimer's disease, before a clinical diagnosis of probable Alzheimer's disease is possible, using $\left[{ }^{18} \mathrm{~F}\right]$ fluorodeoxyglucose positron emission tomography. First, 66 patients with probable Alzheimer's disease with a spectrum of dementia severity (Mini-Mental State Examination score, 0-23) were recruited and studied. Cortical metabolic activity was analyzed topographically using three-dimensional stereotactic surface projections. Regression analysis was performed for each brain pixel to predict metabolic patterns of very early disease. Predictions were tested prospectively in a group of 8 patients who complained only of memory impairment without general cognitive decline (Mini-Mental State Examination score, $25 \pm 1$ ) at the time of scanning but whose condition later progressed to probable Alzheimer's disease. Both results were compared to cerebral metabolic activity in 22 age-similar normal control subjects. Prediction and analysis of actual patients consistently indicated marked metabolic reduction $(21-22 \%)$ in the posterior cingulate cortex and cinguloparietal transitional area in patients with very early Alzheimer's disease. Mean metabolic reduction in the posterior cingulate cortex was significantly greater than that in the lateral neocortices or parahippocampal cortex. The result suggests a functional importance for the posterior cingulate cortex in impairment of learning and memory, which is a feature of very early Alzheimer's disease.

Minoshima S, Giordani B, Berent S, Frey KA, Foster NL, Kuhl DE. Metabolic reduction in the posterior cingulate cortex in very early Alzheimer's disease. Ann Neurol 1997;42:85-94

Energy metabolic abnormalities in the brain of patients with Alzheimer's disease have been investigated using in vivo imaging techniques such as positron emission tomography (PET). Earlier PET reports indicated focal accentuation of metabolic reduction in the parietal and temporal cerebral cortices in addition to general global decline [1-5]. In contrast to the association cortices, energy metabolism in the primary cortices and subcortical structures is preserved relatively when compared to that in the parietotemporal and frontal association cortices. Such structures include the primary sensorimotor and visual cortices, striatum, thalamus, cerebellum, and pons $[2,6]$. Although these findings may not be specific to Alzheimer's dementia [7,8], the stereotypical feature of parietotemporal metabolic reduction is recognized consistently and is of potential utility in the clinical diagnosis of Alzheimer's disease [9, 10].

In addition to neocortical abnormalities in Alzheimer's disease, recent topographical analysis of PET images found significant metabolic reduction in the posterior cingulate cortex in patients with probable
Alzheimer's disease [11], of comparable severity as that in the parietotemporal association cortex. Metabolic reduction in the posterior cingulate cortex coincides with the observation of severe neurodegeneration in moderate to severe Alzheimer's disease reported in early literature [12]. The metabolic abnormality in the posterior cingulate cortex has been previously overlooked in imaging analysis and was reported only occasionally [13]. This seems to be in part due to the difficulty to appreciate exactly the posterior cingulate cortex on twodimensional tomographic slices oriented parallel to the conventional orbitomeatal plane.

Energy metabolic alteration in the parietal and temporal cortices, as measured by PET, may precede certain cognitive symptoms in Alzheimer's disease [14] and can be observed in asymptomatic subjects at risk for familial Alzheimer's disease [15, 16]. However, the time course of metabolic abnormalities observed in the posterior cingulate cortex is largely unknown. Using a PET topographical analysis technique [10], a recent collaborative study demonstrated possible metabolic re-
From the Departments of ${ }^{*}$ Internal Medicine, $†$ Psychiatry, and \$Neurology, The University of Michigan Medical School, Ann Arbor, MI.

Received Mar 25, 1996, and in revised form Nov 22. Accepted for publication Dec 18, 1996.
Address correspondence to Dr Minoshima, Division of Nuclear Medicine, Department of Internal Medicine, The University of Michigan, BIG412 University Hospital, Ann Arbor, MI 48109 0028 . 
duction in the posterior cingulate cortex in apolipoprotein $\mathrm{E}$ type 4 homozygotes without symptoms of dementia [17]. Analysis of pathological changes in very early disease may provide a better understanding of the primary disease processes without secondary or reactive changes that may complicate the advanced stages of chronic illness. In addition, such knowledge may help develop more sensitive and specific diagnostic schemes. Investigations based on neuropathological examinations, however, may not be able to address easily early disease processes because of the difficulty in obtaining brain specimens from patients diagnosed to have a very early stage of the disease. In vivo imaging potentially has advantages in such situations because of its noninvasive nature.

In this study, we investigated the topographical distribution of cerebral energy metabolic alterations in Alzheimer's disease, particularly at a very early stage. We examined cerebral metabolic PET data sets obtained from two different populations with Alzheimer's disease. First, we predicted a metabolic pattern of a very early stage of the disease using regression analysis of cerebral metabolism and mental status test results in probable Alzheimer's disease patients with dementia of various severities. Second, we confirmed the predicted result in actual patients with very early Alzheimer's disease whose diagnosis was established later in a clinical follow-up or at autopsy.

\section{Materials and Methods}

\section{Subjects}

Sixty-six patients with probable Alzheimer's disease were recruited from the Department of Neurology Cognitive Disorders Clinic at The University of Michigan Medical Center between 1989 and 1994. The recruitment was part of an ongoing, cross-sectional study of Alzheimer's disease and intended to involve a spectrum of dementia severity. The clinical diagnosis of probable Alzheimer's disease was based on National Institute of Neurological and Communicative Disorders and Stroke-Alzheimer's Disease and Related Disorders Association (NINCDS-ADRDA) criteria [18]. The mean age of this group was $68 \pm 7.3$ (mean \pm standard deviation), and the range from 50 to 82 years. Thirty-one men and 35 women were included. Seventeen patients were confirmed to be postmenopausal by medical chart review. Two patients were on estrogen replacement prior to or immediately after the scan data were obtained. At the time of PET scanning, the mean severity of general cognitive decline measured as a Mini-Mental State Examination (MMSE) score [19] was $15 \pm 6.0$, ranging from 0 to 23 . The clinical dementia rating (CDR) [20] was 0.5 (questionable) in 5 patients, 1 (mild) in 39 patients, 2 (moderate) in 14 patients, and 3 (severe) in 8 patients.

Twenty-three patients who complained of only memory impairment without general cognitive decline were recruited from the same clinic and partially by advertising between 1989 and 1993. All patients underwent thorough neuropsychological testing that revealed quantified, objective evidence of memory impairment with no apparent loss in general cognitive, behavioral, or functional status. The classification of memory impairment required normal mental status (MMSE scores $\geq 24, \mathrm{CDR}=0.5$ ) with memory performance falling one standard deviation below the normal reference, that is, a combination score of Wechsler Memory Scale (WMS) subtests IV (Logical Memory) and VII (Paired Associates Learning) of 19 or less [21], and/or a score on the Benton Visual Retention Test of 6 or lower [22]. Each patient underwent PET at the time of the initial evaluation and was followed clinically for at least 2 years. Eight of 23 patients satisfied the diagnostic criteria of probable Alzheimer's disease during the follow-up period. The interval berween the collection of scan data and the diagnosis of probable Alzheimer's disease averaged 23 months, ranging from 4 to 39 months. These patients included 5 men and 3 women with an average age of $69 \pm 4.6$ years and a range from 63 to 73 years. All women were postmenopausal, and 1 patient was on estrogen replacement prior to or immediately after the scanning. At the time of PET, the mean MMSE score was $25 \pm$ 1.1, which declined to $20 \pm 3.0$ during the follow -up period. Despite a mild decline in general mental status, severe learning and memory impairment was evident in these $8 \mathrm{pa}^{-}$ tients at the time of PET. For example, of a possible score of 23 on WMS subtest IV, patients scored $3.5 \pm 1.5$ on immediate recall and only $0.17 \pm 0.25$ on 30 -minute delayed recall. One patient died approximately 5 years after the initial evaluation, and neuropathological examination including silver, myelin, glial, and ubiquitin stains confirmed Alzheimer's disease pathology without coexistent disorders. In the following sections, these patients are referred to as having "very early Alzheimer's disease."

Twenty-two age-similar normal control subjects were recruited by advercising. This group included 7 men and 15 women with an average age of $64 \pm 7.5$ years and a range from 52 to 76 . The mean MMSE score was $28 \pm 1.0$, ranging from 26 to 30 . The CDR was 0 in all subjects.

\section{Cerebral Glucose Metabolic Measurement}

Each patient underwent cerebral metabolic measurement using PET. The protocol was approved by the Institutional Review Board of The University of Michigan Medical School. Written informed consent was obtained from each subject. A Siemens ECAT scanner (model 931/08-12, CTI, Knoxville, TN), which collects 15 simultaneous slices with $6.75-\mathrm{mm}$ slice separation, was used. All patients were fasted for at least 4 hours prior to scanning. Ten millicuries of $2-\left[{ }^{18} \mathrm{~F}\right]$ fluoro2-deoxy-D-glucose was administered intravenously while a patient was kept in a dimly lit room. A set of transaxial images was obtained starting at 30 minutes following the injection. Images were reconstructed using a Shepp filter (cutoff frequency, 0.3 cycle/projection element) and calculated attenuation correction, giving a reconstructed image resolution of approximately $8-\mathrm{mm}$ full width at half maximum.

PET image analysis was performed by fully automated methods, thus minimizing subjective bias and variability. Each image set was realigned to the bicommissure stereotactic coordinate system [23]. The difference in an individual's brain size was removed by linear scaling, and regional anatomical differences were minimized by a nonlinear warping 
technique [24]. As a result, each brain was standardized anatomically to match with a standard atlas brain [23] while preserving regional metabolic activity. Subsequently, maximum cortical activity was extracted to adjacent predefined surface pixels on a pixel-by-pixel basis using a threedimensional stereotactic surface projection technique (3DSSP) [10]. This cortical data extraction technique compensates for small anatomical differences in gray matter structures (such as variable depth of gyri) across patients and minimizes a partial volume of the white matter. Approximately 16,000 predefined surface pixels covered the entire cortex, including the medial aspect of the two hemispheres. The extracted data on cortical activity were used in the following analyses.

\section{Data Analysis}

Data sets were normalized to the pontine activity prior to further analyses, as energy metabolic activity in the pons is relatively preserved in Alzheimer's disease [6]. Normalization not only enables data analysis of image sets without absolute quantification but also reduces global metabolic variation across subjects, and thus increases sensitivity of the analysis.

Prior to data analysis, gender differences in metabolic activity were examined in 66 patients with probable Alzheimer's disease. The mean ages of the men and women were $67 \pm 8$ and $69 \pm 7$ years, respectively. The mean ages at onset in the men and women were $64 \pm 9$ and $65 \pm 7$ years, respectively. The mean disease durations in the men and women were $3.9 \pm 3.0$ and $3.8 \pm 2.5$ years, respectively. The mean MMSE scores were $15 \pm 6$ and $15 \pm 6$, respectively. The mean CDRs were $1.3 \pm 0.7$ and $1.5 \pm$ 0.8 , respectively. These data suggest that the two gender groups were similar for age, onset age, disease duration, and severity of dementia. When regional metabolic activity in these two groups was examined, none of the lateral association cortices, cingulate cortices, or medial temporal cortex showed statistically significant differences (two-sample Student's $t$ test). We therefore pooled the two groups in the further analyses.

With data sets of 66 patients with probable Alzheimer's disease, regression analysis was performed to predict the metabolic pattern of very early Alzheimer's disease. For each extracted cortical pixel, linear regression was calculated using metabolic activities and MMSE scores for the 66 patients. Predicted metabolic activity at an MMSE score of 30 was then extrapolated based on an estimated regression line, resulting in a three-dimensional metabolic pattern of very early Alzheimer's disease. In addition, metabolic patterns at MMSE scores of 20,10, and 0 were generated to examine progression of the disease. Note that the pattern at an MMSE score of 30 was extrapolated from patient data of MMSE scores 0 to 23 to represent a hypothetical metabolic pattern of very early Alzheimer's disease, and did not indicate a metabolic pattern in actual patients with an MMSE score of 30 . These patterns were compared to those in 22 normal subjects using pixel-by-pixel Z-score analysis ([normal mean - predicted value]/normal standard deviation). By using $\mathrm{Z}$-score analysis, the magnitude of metabolic reduction across areas with different baseline (normal) metabolic activity could be compared. Peak metabolic reduction in the pre- dicted very early Alzheimer's disease was determined stereotactically.

The metabolic pattern of very early Alzheimer's disease was confirmed further using data sets obtained from 8 patients with only memory impairment who were diagnosed later as having probable Alzheimer's disease. The extracted cortical activity of these patients was compared to that of 22 normal subjects using a two-sample Student's $t$ test on a pixel-by-pixel basis. Calculated $t$ values were converted to $\mathrm{Z}$ values using a probability integral transformation for comparison with the above regression analysis. A statistical significance threshold for $p=0.05$ was calculated on the 3DSSP Z map using a "unified" formula which controls multiple pixel comparison and a shape of the stochastic process on the 3D-SSP format [25]. Regional metabolic reduction in this patient group was determined stereotactically in three dimensions.

To examine individual data in patients with very early Alzheimer's disease, region-of-interest analysis was performed in addition to the above pixel-by-pixel analysis. Five regions of interest were predefined stereotactically for each hemisphere based on the definition in the stereotactic brain atlas [23] as follows: parietal association cortex including Brodmann's cortical areas 5,7,39, and 40; temporal association cortex including areas $21,22,37$, and 38 ; frontal association cortex including areas $6,8,9,10,11,44,45,46$, and 47 ; parahippocampal gyrus including areas 27,28 , and 34 ; and posterior cingulate cortex including areas 23 and 31 . Although these cytoarchitectural areas cannot be appreciated directly on PET images, we use this numbering system to describe objectively regions included in the analysis. In addition, two regions of interest were obtained as a reference: anterior cingulate cortex including areas 24 and 32 and primary visual cortex including area 17 . The extracted cortical activity was averaged within these regions. Since hemispheric asymmetry in metabolic alteration may exist in Alzheimer's disease [3], hemispheres were analyzed independently. Each regional value for a patient was compared to a cortesponding mean value of 22 normal subjects on a region-by-region basis, and the magnitude of metabolic reduction was expressed as a $Z$ score. A Student's $t$ test was used to assess differences in region-of-interest values between normal subjects and patients with very early Alzheimer's disease. A Wilcoxon signed rank test was used to assess differences in the magnitude of metabolic reduction between the posterior cingulate cortex and other regions.

\section{Results}

In normal subjects, there was intense energy metabolic activity in the posterior cingulate cortex and cinguloparietal transitional area (Fig 1). Metabolic activity in the posterior cingulate cortex (right, $1.46 \pm 0.123$; left, $1.43 \pm 0.112$, relative to pontine activity) was almost as high as that in the primary visual cortex (right, $1.55 \pm 0.148$; left, $1.51 \pm 0.134$ ) and significantly higher $\left(p<0.01 \times 10^{-8}\right.$, one-sample $t$ test $)$ than that in the anterior cingulate cortex (right, $1.24 \pm 0.107$; left, $1.23 \pm 0.0994$ ).

A predicted metabolic pattern of Alzheimer's disease 
Fig 1. Predicted patterns of cerebral energy metabolism in Alzheimer's disease at Mini-Mental State Examination (MMSE) scores of 30, 20, 10 , and $O$ in comparison to averaged metabolic activity of age-similar normal control subjects (N). Predicted metabolic activity at MMSE of 30 is extrapolated from patients with MMSE scores of $O$ to 23 using regression analysis and does not represent a metabolic pattern of actual patients with an MMSE score of 30 . Cerebral metabolic activity is normalized to the pontine activity (norm CMRglc). Images represent right lateral (RT.LAT), left lateral (LT.LAT), right medial (RT.MED), and left medial (LT.MED) views of the brain. Metabolic activity in the posterior cingulate cortex and the cinguloparietal transitional area is demonstrated on the right and left medial views (arrows on the left medial view of $N)$. The activity in these regions decreases markedly at the MMSE score of 30 . Metabolic reduction in the lateral neocortex becomes obvious when the disease progresses. Note the relatively preserved metabolism in the primary sensorimotor cortex, occipital cortex, and cerebellum.

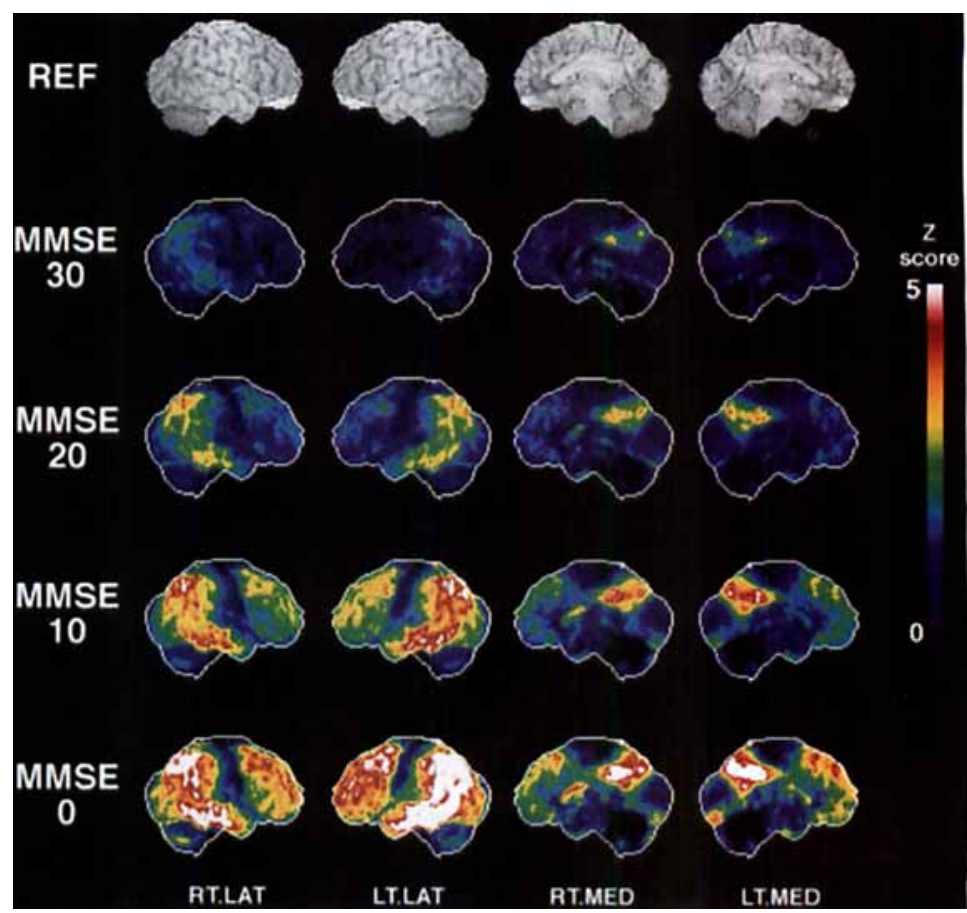

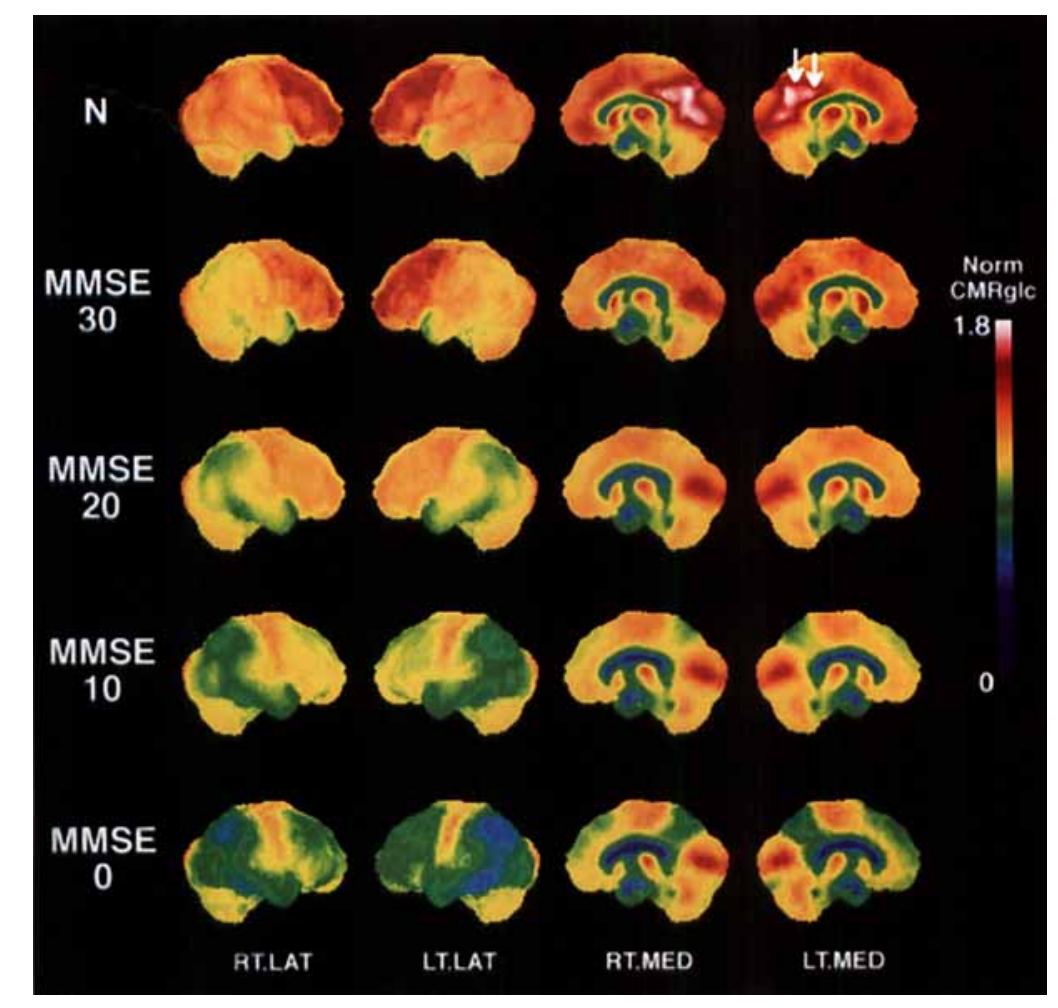

Fig 2. Z-score images of predicted metabolic reduction in Alzheimer's disease at Mini-Mental State Examination (MMSE) scores of 30, 20, 10, and 0 . Higher pixel intensity represents greater metabolic reduction. The first row represents an anatomical reference (REF) for visual inspection. Images represent right lateral (RT.LAT), left lateral (LT.LAT), right medial (RT.MED), and left medial

(LT. MED) views of the brain. Topographical distributions of metabolic reduction with various dementia severities are evident on these images. The right and left medial views at an MMSE score of 30 reveal very early metabolic reduction in the posterior cingulate cortex extending to the cinguloparietal transitional area. Metabolic reduction in the lateral neocortex is present at an MMSE score of 30 , but is relatively mild in comparison to the posterior cingulate cortex. Metabolic activity in the anterior cingulate cortex is relatively preserved. 
Table. Locations of Peak Metabolic Reduction in Very Early Alzheimer's Disease ${ }^{\text {a }}$

\begin{tabular}{|c|c|c|c|c|}
\hline & Posterior Cingulate & Cinguloparietal & Parietal & Temporal \\
\hline \multicolumn{5}{|l|}{ Right hemisphere } \\
\hline Predicted (MMSE score of 30) & $(0,-31,29)$ & $(0,-67,36)$ & $(42,-60,47)$ & $(60,-31,-18)$ \\
\hline Actual patients & $(0,-38,29)$ & $(0,-63,34)$ & $(35,-65,50)$ & $(60,-40,-18)$ \\
\hline \multicolumn{5}{|l|}{ Left hemisphere } \\
\hline Predicted (MMSE score of 30 ) & $(0,-31,29)$ & $(0,-69,36)$ & $(46,-60,47)$ & $(53,-33,-25)$ \\
\hline Actual patients & $(0,-36,32)$ & $(0,-65,34)$ & $(48,-67,38)$ & $(60,-31,-16)$ \\
\hline
\end{tabular}

The coordinates $(x, y, z)$ represent midlateral, posterior $(-)$ or anterior $(+)$, and ventral $(-)$ or dorsal $(+)$ coordinates of the brain, respectively, in a stereotactic millimetric scale [23]. Peak $x$ coordinates of the posterior cingulate cortex and cinguloparietal transitional area were projected to the midsagittal plane $(x=0)$.

MMSE $=$ Mini-Mental State Examination.

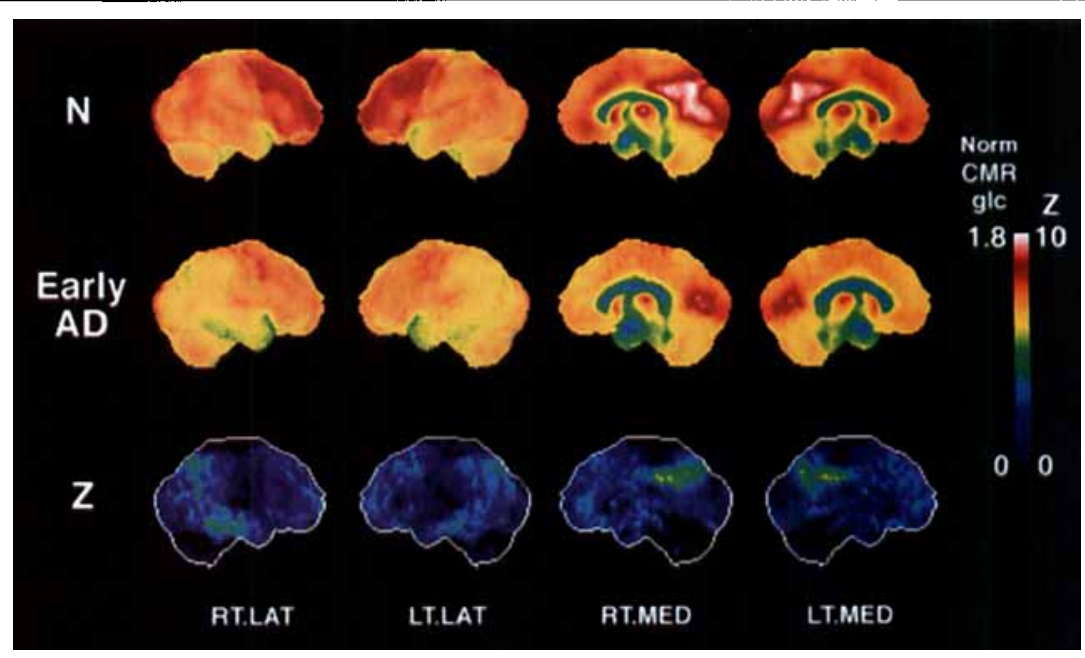

Fig 3. Cerebral metabolic activity in patients with very early Alzheimer's disease (Early AD) in comparison to that of normal controls (N, identical to $N$ in Fig $I)$. Two group-averaged images were compared using $\mathrm{t}$ statistics and converted to $Z$ images ( $Z$ ). Images represent right lateral (RT.LAT), left lateral (LT.LAT), right medial (RT.MED), and left medial (LT.MED) views of the brain. Metabolic reduction in the posterior cingulate cortex and the cinguloparietal area is demonstrated clearly on both groupaveraged images (Early $A D$ ) and $Z$ images ( $Z$ ). Relatively mild but definite metabolic reduction in the parietal and temporal association cortices was also evident in very early Alzheimer's disease. Note the consistency of these results with the predicted pattern of very early Alzheimer's disease (see Fig 2).

at an MMSE score of 30, which was extrapolated from data sets of MMSE scores of 0 to 23, revealed the disappearance of such intense metabolic activity in the posterior cingulate correx (see Fig 1). Predicted metabolic activity in the parietal and temporal association cortices showed only mild reduction at this stage. Areas of predicted metabolic reduction were clearly demonstrated on Z-score maps (Fig 2), and peaks of metabolic reduction were localized stereotactically (Table). Focally accentuated metabolic reduction in the posterior cingulate cortex was evident bilaterally. As the disease progresses, metabolic reduction in the lateral neocortex becomes more apparent. At MMSE scores of 10 and 0 , there is metabolic reduction involving nearly the entire association cortex but sparing relatively the primary sensorimotor cortex, primary visual cortex, and cerebellum (Figs 1,2). This pattern is consistent with a well-known metabolic feature of Alzheimer's disease.

The metabolic pattern actually observed in patients with very early Alzheimer's disease is mostly consistent with the above-described predicted pattern. In these patients with very early Alzheimer's disease, there was significant metabolic reduction in the posterior cingulate cortex extending to the cinguloparietal transitional area (Fig 3). Peak metabolic reduction was in close agreement with the predicted result (see Table). Z scores of peak metabolic reduction in the posterior cingulate cortex were 5.72 for the right and 5.53 for the left. Peak $\mathrm{Z}$ scores in the cinguloparietal transitional area were 5.29 for the right and 5.43 for the left. All peaks exceeded the statistical threshold of $Z=4.53$. Peak metabolic reduction in the lateral association cor- 

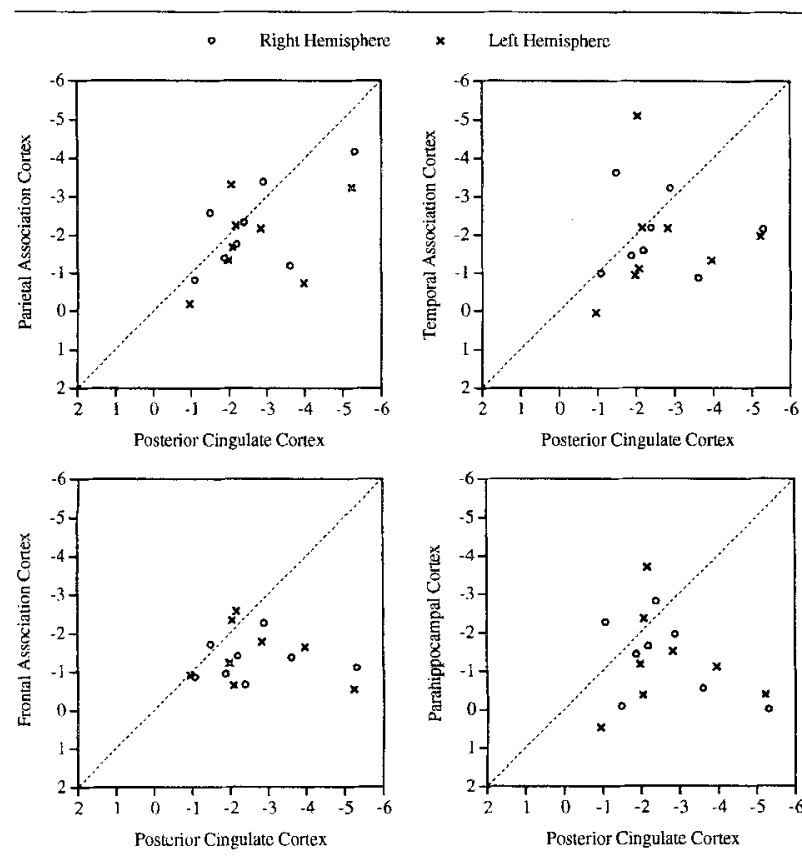

Fig 4. Individual analysis of cerebral metabolic reduction in patients with very early Alzheimer's disease. $Z$ scores representing the magnitude of metabolic reduction in the parietal, temporal, and frontal association cortices and parahippocampal cortex are compared to those in the posterior cingulate cortex. The right and left hemispheres are analyzed independently. The diagonal dotted lines represent the line of identity. Data points toward the right-lower corner indicate a reduction in the posterior cingulate greater than that in other cortical areas. In each comparison, most data points fall into the right-lower half of the graph, suggesting relatively severe metabolic reduction in the posterior cingulate cortex in comparison to other cortical areas.

tices was less significant, and only the right parietal and temporal association cortices $(\mathrm{Z}=4.72$ and 4.91 , respectively) exceeded the statistical threshold. Compared to normal controls, and based on region-of-interest analysis, metabolic reductions in the right and left posterior cingulate cortices were $22 \%$ and $21 \%$, respectively (Student's $t$ test, $p=0.0007$ ). In contrast to the posterior cingulate cortex, metabolic reduction in the anterior cingulate cortex was mild.

When patients with very early Alzheimer's disease were examined individually, metabolic reduction in the posterior cingulate cortex was significantly greater than that in the other cortical regions (Fig 4). In comparison to parietal, temporal, and frontal association cortices, the greater metabolic reduction in the posterior cingulate cortex was significant at $p=0.070,0.049$, and 0.004 , respectively (Wilcoxon test, two-tailed probability using normal approximation). Although the posterior cingulate cortex showed the greatest metabolic reduction, mild metabolic reduction in the parietal and temporal cortices also existed in very early Alz- heimer's disease (see Figs 3, 4). The metabolic reduction in the posterior cingulate cortex was also significantly greater than that of the parahippocampal cortex $(p=0.015)$.

\section{Discussion}

Topographical analysis of $\left[{ }^{18} \mathrm{~F}\right]$ fluorodeoxyglucose PET revealed severe and consistent metabolic reduction in the posterior cingulate cortex in very early Alzheimer's disease. This finding may provide an additional important clue to the pathology of early Alzheimer's disease and needs to be interpreted in the context of pathological and neurochemical alterations observed in Alzheimer's disease as well as cortical neuronal connections.

Brun and Gustafson [12] reported severe degeneration in the posterior cingulate cortex in patients with Alzheimer's disease. One of 2 mildly demented patients, however, did not demonstrate severe neurodegeneration in the posterior cingulate cortex or in the other association cortices [26]. Subsequently, detailed neuropathological examination [27] demonstrated various patterns of laminar degeneration in the posterior cingulate cortex. In their series, $12 \%$ of patients revealed no neuronal loss in the posterior cingulate cortex, although as the authors discussed, the removal of cortical afferents could have indirectly caused increasing neuron density. The topographical distribution of neurofibrillary tangles is not necessarily more severe in the posterior cingulate cortex compared to the medial and inferior temporal cortex [28]. Pathological examination of presumably very early Alzheimer's disease [29] revealed extensive neurofibrillary tangle formation only in the medial and inferior temporal cortex, but not in the posterior cingulate cortex. This pathological evidence summarizes the presence of significant neurodegeneration in the posterior cingulate cortex, which, however, may not be the most consistent structural change in very early Alzheimer's disease. The discordance between our current findings of very early metabolic reduction in the posterior cingulate cortex and the pathological evidence raises the possibility of functionally, but not structurally altered neuronal circuits in the posterior cingulate cortex. Simonian and Hyman [30] demonstrated the absence of correlation between structural pathology and functional activity of neurons measured by cytochrome oxidase activity, suggesting that functional alteration may precede or disagree with structural abnormality in Alzheimer's disease. In addition, reduced cytochrome oxidase activity occurs experimentally following deafferentation of neuronal circuits [31] due to a decrease in neuronal firing and $\mathrm{Na} / \mathrm{K}$ ATPase activity [32]. Deoxyglucose uptake is known to correlate with neuronal activity, particularly at synapses instead of perikarya [33], and to coincide with cytochrome oxidase staining [34]. Therefore, our findings of very early metabolic reduction in the posterior cin- 
gulate cortex could be explained by very early functional or structural deafferentation, or both, caused by primary neural degeneration in the other parts of the brain in addition to subsequent or simultaneous primary degeneration in the posterior cingulate cortex.

As indicated by deafferentation, neural connection seems to play an important role in the development of Alzheimer's pathology [35]. Parts of the brain affected in Alzheimer's disease are tightly interconnected. In light of connectivity, the posterior cingulate cortex has various connections with other limbic structures as well as association cortices. These connections and the cortical cytoarchitecture of the posterior cingulate cortex are distinct from those of the anterior cingulate cortex [36]. Afferent pathways to the posterior cingulate cortex studied in monkeys [36] include the contralateral posterior cingulate cortex, parietal association cortex (area 7), superior temporal cortex (area 22), ventromedial temporal cortex including areas medial to the occipitotemporal sulcus, entorhinal cortex (area 28), and perirhinal cortex (area 35), vicinity of the principal sulcus (area 46), and other frontal cortices (areas 8, 9, 10, and 11). Compared to primary cortices, these structures are affected severely in Alzheimer's disease. There are also afferent pathways to the posterior cingulate cortex directly from the entorhinal/perirhinal cortices $[36,37]$ where Alzheimer's pathology was suggested to occur initially $[29,38,39]$. In contrast to entorhinal cortex layer II, which forms the perforant pathway to the hippocampal formation, layer IV of the entorhinal and posterior parahippocampal cortices gives widespread projections to sensory-specific and multimodal association cortices [40-42]. In Alzheimer's disease, it is reported that layers II and IV are affected severely compared to other layers [43].

The severe metabolic reduction in the posterior cingulate cortex coincides with previous observations of neuronal degeneration in the posterior cingulate/ retrosplenial cortex induced by $N$-methyl-D-aspartate (NMDA) antagonists such as phencyclidine, MK-801, and ketamine $[44,45]$. The mechanism is attributed to the abolishment of gamma-aminobutyric acid (GABA) inhibition, which is maintained by tonic glutamatergic input, and thus major glutamatergic and cholinergic inputs to cortical neurons induce excitotoxicity [46]. Since systemically administered NMDA antagonists act globally, the posterior cingulate cortex seems to be the structure that demonstrates the initial evidence of global deterioration of the glutamatergic neuronal transmission. Despite difficulties in determining the glutamatergic neurotransmitter system in the brain, there is accumulating evidence that corticocortical fibers of pyramidal neurons are glutamatergic $[47,48]$, and those glutamatergic neurons are degenerating substantially in Alzheimer's disease [49-52]. Considering the above-cited evidence, the early metabolic reduction in the posterior cingulate cortex observed in the current study may be a consequence of global derangement of cortical glutamatergic neurons which interconnect the posterior cingulate cortex and other cortical structures, including the entorhinal cortex, as discussed previously. In fact, the parietal and temporal association cortices had a relatively mild but not negligible metabolic reduction in patients with very early Alzheimer's disease (see Fig 3).

A somewhat puzzling result is the relatively mild metabolic reduction in the parahippocampal cortex. This result is in agreement with previous observations using an ultra-high-resolution PET scanner [53] and $\left[{ }^{11} \mathrm{C}\right]$ deoxyglucose [13]. A technical problem in measuring metabolic activity in a relatively small area of the medial and inferior temporal lobe is always a concern. Using exactly the same analytical technique, however, we previously demonstrated severe metabolic reduction in the medial temporal lobe in patients with progressive limbic sclerosis [54]. The presence of atrophy affects PET measurement of regional metabolic activity owing to partial volume effects, but in a way metabolic activity appears more decreased instead of relatively preserved. When a presynaptic terminal density in Alzheimer's disease was examined, the most severe loss was found in the frontal and parietal cortices, not in the entorhinal cortex or hippocampus [55]. Synaptic density in entorhinal cortex layers III and V was reported to be preserved [56]. Since deoxyglucose uptake correlates with synaptic activity [33], relatively mild energy metabolic reduction in the parahippocampal cortex seems to be in agreement with the structural observations. A relatively mild reduction in synaptic activity in contrast to the severe neuronal degeneration in the medial temporal lobe could be, at least in part, attributable to synaptic plasticity. Following an experimental lesion in the entorhinal cortex, synaptic regeneration in the dentate gyrus was suggested [57]. There is morphological evidence that the remaining synapses in the dentate gyrus of Alzheimer's disease patients show an increase in synaptic junctional area [58]. Furthermore, after loss of entorhinal neurons in $\mathrm{Alz}_{-}$ heimer's disease, cholinergic and commissural associational sprouting seems to take place in the hippocampal formation [59, 60]. The relationship among synaptic plasticity and regional energy metabolism, however, needs to be investigated further in patients with Alzheimer's disease.

Neuropsychological studies suggest the role of the posterior cingulate cortex in spatial learning and memory in monkeys [61]; in acquisition and long-term memory processes in rats [62]; in learning and memory deficits in mice due to posterior, but not anterior, cingulate lesions [63]; in spatial orientation and memory in rats and monkeys [64]; and in verbal and auditory memory in monkeys [65]. In addition, the retrosplenial 
cortex is involved in anterograde and retrograde amnesia in humans [66] and visual tracking tasks with a delay in monkeys [67], although some of these observations may be attributable in part to injury to the cingulum [68]. As indicated in the current study, early metabolic reduction in the posterior cingulate cortex is associated with severe learning and memory impairment without general cognitive decline. Comparisons between metabolic alterations in the posterior cingulate cortex and detailed neuropsychological test results are subject to further investigations.

In our previous report of normal subjects and multiinfarct patients, we did not observe metabolic abnormalities in the posterior cingulate cortex $[10,69]$. However, subsequent analysis of clinically diagnosed Parkinson's disease with dementia [70] and autopsyproved diffuse Lewy body disease [8] demonstrated metabolic reduction in the posterior cingulate cortex similar to that observed in the current study. Such similarity in posterior cingulate abnormality may suggest the involvement of common pathophysiological processes in interrelated neurodegenerative diseases. Nevertheless, these findings suggest a functional importance of the posterior cingulate cortex in a part of learning and memory network systems.

In summary, topographical analysis of energy metabolism in Alzheimer's disease was conducted using $\left[{ }^{18} \mathrm{~F}\right]$ fluorodeoxyglucose PET and three-dimensional image analysis. Despite pathological evidence of early involvement of the entorhinal cortex in Alzheimer's disease, the posterior cingulate cortex shows severe functional reduction in a very early stage of the disease. Implications of this finding relating to cortical anatomy and pathological and neurochemical changes in Alzheimer's disease are discussed.

This study was supported in part by RO1-NS24896 from the National Institutes of Health, DE-FG02-87-ER60561 from the US Department of Energy, P50-AG08671 from the National Institutes of Health (the Michigan Alzheimer's Disease Research Center), and P30-AG08808 from the National Institutes of Health (The University of Michigan Claude D. Pepper Older American Independence Center).

The authors thank Sid Gilman, MD, for his continuous support, Robert A. Koeppe, PhD, for his assistance in collecting PET data, Nancy Lowenbergh, BSN, for recruiting subjects and maintaining a patient scan database, Laurie A. Bluemlein, MS, RN, for medical chart review, PET technologists for their skillful performance in data acquisition, and cyclotron operators and chemists for their production of radiopharmaceuticals.

\section{References}

1. Frackowiak RS, Pozzilli C, Legg NJ, et al. Regional cerebral oxygen supply and utilization in dementia. A clinical and physiological study with oxygen-15 and positron tomography. Brain 1981;104:753-778

2. Benson DF, Kuhl DE, Hawkins RA, et al. The fluorodeoxyglu- cose ${ }^{18} \mathrm{~F}$ scan in Alzheimer's disease and multi-infarct dementia. Arch Neurol 1983;40:711-714

3. Foster NL, Chase TN, Fedio P, et al. Alzheimer's disease: focal cortical changes shown by positron emission tomography. Neurology 1983;33:961-965

4. Friedland RP, Budinger TF, Ganz E, et al. Regional cerebral metabolic alterations in dementia of the Alzheimer type: positron emission tomography with $\left[{ }^{18} \mathrm{~F}\right]$ fluorodeoxyglucose. J Comput Assist Tomogr 1983;7:590-598

5. Kuhl DE. Imaging local brain function with emission computed tomography. Radiology 1984;150:625-631

6. Minoshima S, Frey KA, Foster NL, Kuhl DE. Preserved pontine glucose metabolism in Alzheimer disease: a reference region for functional brain image (PET) analysis. J Comput Assist Tomogr 1995;19:541-547

7. Schapiro MB, Pietrini P, Grady CL, et al. Reductions in parietal and temporal cerebral metabolic rates for glucose are not specific for Alzheimer's disease. J Neurol Neurosurg Psychiatry 1993;56:859-864

8. Albin RL, Minoshima S, D'Amato CJ, et al. Fluorodeoxyglucose positron emission tomography in diffuse Lewy body disease. Neurology 1996;47:462-466

9. Herholz K, Adams R, Kessler J, et al. Criteria for the diagnosis of Alzheimer's disease with positron emission tomography. Dementia 1990;1:156-164

10. Minoshima S, Frey KA, Koeppe RA, et al. A diagnostic approach in Alzheimer's disease using three-dimensional stereotactic surface projections of fluorine-18-FDG PET. J Nucl Med 1995;36:1238-1248

11. Minoshima S, Foster NL, Kuhl DE. Posterior cingulate cortex in Alzheimer's disease. Lancet 1994;344:895

12. Brun A, Gustafson L. Distribution of cerebral degeneration in Alzheimer's disease. A clinico-pathological study. Arch Psychiatr Nervenkr 1976;223:15-33

13. Nyback $H$, Nyman $H$, Blomqvist $G$, et al. Brain metabolism in Alzheimer's dementia: studies of " $\mathrm{C}$-deoxyglucose accumulation, CSF monoamine metabolites and neuropsychological test performance in patients and healthy subjects. J Neurol Neurosurg Psychiatry 1991;54:672-678

14. Haxby JV, Grady CL, Duara R, et al. Neocortical metabolic abnormalities precede nonmemory cognitive defects in early Alzheimer's-type dementia. Arch Neurol 1986;43:882-885

15. Kennedy AM, Frackowiak RS, Newman SK, et al. Deficits in cerebral glucose metabolism demonstrated by positron emission tomography in individuals at risk of familial Alzheimer's disease. Neurosci Lett 1995;186:17-20

16. Small GW, Mazziotta JC, Collins MT, et al. Apolipoprotein E type 4 allele and cerebral glucose metabolism in relatives at risk for familial Alzheimer disease. JAMA 1995;273:942-947

17. Reiman EM, Caselli RJ, Yun LS, et al. Preclinical evidence of Alzheimer's disease in persons homozygous for the epsilon 4 allele for apolipoprotein E. N Engl J Med 1996;334:752-758

18. McKhann G, Drachman D, Folstein $M$, et al. Clinical diagnosis of Alzheimer's disease: report of the NINCDS-ADRDA Work Group under the auspices of Department of Health and Human Services Task Force on Alzheimer's disease. Neurology 1984;34:939-944

19. Folstein MF, Folstein SE, McHugh PR. "Mini-mental state." A practical method for grading the cognitive state of patients for the clinician. J Psychiatr Res 1975;12:189-198

20. Gelb DJ, St. Laurent RT. Alternative calculation of the global clinical dementia rating. Alzheimer Dis Assoc Disord 1993;7: 202-211

21. Wechsler D. Wechsler Adult Intelligence Scale. New York: Psychological Corporation, 1955

22. Benton AL. The Revised Visual Retention Test. 4th ed. New York: Psychological Corporation, 1974 
23. Talairach J, Tournoux P. Co-planar stereotaxic atlas of the human brain. New York: Thieme, 1988

24. Minoshima S, Koeppe RA, Frey KA, Kuhl DE. Anatomic standardization: linear scaling and nonlinear warping of functional brain images. J Nud Med 1994;35:1528-1537

25. Worsley KJ, Marrett S, Neelin P, Evans AC. A unified statistical approach for determining significant signals in location and scale space images of cerebral activation. In: Myers R, Cunningham V, Baily $D$, et al, eds. Quantification of brain function using PET. San Diego: Academic, 1996:327-333

26. Brun A, Englund E. Regional pattern of degeneration in $\mathrm{Alz}_{-}$ heimer's disease: neuronal loss and histopathological grading. Histopathology 1981;5:549-564

27. Vogt BA, Van Hoesen GW, Vogt LJ. Laminar distribution of neuron degeneration in posterior cingulate cortex in Alzheimer's disease. Acta Neuropathol (Berl) 1990;80:581-589

28. Arnold SE, Hyman BT, Flory J, et al. The topographical and neuroanatomical distribution of neurofibrillary tangles and neuritic plaques in the cerebral cortex of patients with Alzheimer's disease. Cereb Cortex 1991;1:103-116

29. Hof PR, Bierer LM, Perl DP, et al. Evidence for early vulnerability of the medial and inferior aspects of the temporal lobe in an 82-year-old patient with preclinical signs of dementia. Regional and laminar distribution of neurofibrillary tangles and senile plaques. Arch Neurol 1992;49:946-953

30. Simonian NA, Hyman BT. Functional alterations in neural ciraits in Alzheimer's disease. Neurobiol Aging 1995;16:305-309

31. Borowsky IW, Collins RC. Histochemical changes in enzymes of energy metabolism in the dentate gyrus accompany deafferentation and synaptic reorganization. Neuroscience 1989;33: 253-262

32. Wong-Riley MT. Cytochrome oxidase: an endogenous metabolic marker for neuronal activity. Trends Neurosci 1989;12: 94-101

33. Kadekaro M, Crane AM, Sokoloff L. Differential effects of electrical stimulation of sciatic nerve on metabolic activity in spinal cord and dorsal root ganglion in the rat. Proc Natl Acad Sci USA $1985 ; 82: 6010-6013$

34. Humphrey AL, Hendrickson AE. Background and stimulusinduced patterns of high metabolic activity in the visual cortex (area 17) of the squirrel and macaque monkey. J Neurosci 1983;3:345-358

35. Pearson RC, Esiri MM, Hiorns RW, et ai. Anatomical correlates of the distribution of the pathological changes in the neocortex in Alzheimer disease. Proc Natl Acad Sci USA 1985;82: 4531-4534

36. Baleydier C, Mauguiere F. The duality of the cingulate gyrus in monkey. Neuroanatomical study and functional hypothesis. Brain 1980; 103:525-554

37. Vogt BA, Pandya DN. Cingulate cortex of the rhesus monkey: II. Cortical afferents. J Comp Neurol 1987;262:271-289

38. Braak H, Braak E. Neuropathological staging of Alzheimerrelated changes. Acta Neuropathol (Berl) 1991;82:239-259

39. Arriagada PV, Marzloff K, Hyman BT. Distribution of Alzheimer-type pathologic changes in nondemented elderly individuals matches the pattern in Alzheimer's disease. Neurology 1992;42:1681-1688

40. Kosel KC, Van Hoesen GW, Rosene DL. Non-hippocampal cortical projections from the entorhinal cortex in the rat and rhesus monkey. Brain Res 1982;244:201-213

41. Van Hoesen GW. The parahippocampal gyrus. New observations regarding its cortical connections in the monkey. Trends Neurosci 1982;5:345-350

42. Van Hoesen GW, Hyman BT, Damasio AR. Entorhinal cortex pathology in Alzheimer's disease. Hippocampus 1991;1:1-8

43. Hyman BT, Van Horsen GW, Damasio AR, Barnes CL. Alz- heimer's disease: cell-specific pathology isolates the hippocarnpal formation. Science 1984;225:1168-1170

44. Olney JW, Labruyere J, Price MT. Pathological changes induced in cerebrocortical neurons by phencyclidine and related drugs. Science 1989;244:1360-1362

45. Sharp FR, Jasper P, Hall J, et al. MK-801 and ketamine induce heat shock protein HSP72 in injured neurons in posterior cingulate and retrosplenial cortex. Ann Neurol 1991;30:801-809

46. Olney JW, Labruyere J, Wang G, et al. NMDA antagonist neurotoxicity: mechanism and prevention. Science 1991;254: $1515-1518$

47. Peinado JM, Mora F. Glutamic acid as a putative transmitter of the interhemispheric corticocortical connections in the rat. J Neurochem 1986;47:1598-1603

48. Conti F, Fabri M, Manzoni T. Glutamate-positive corticocortical neurons in the somatic sensory areas I and II of cats. J Neurosci 1988;8:2948-2960

49. Hardy J, Cowburn R, Barton A, et al. Region-specific loss of glutamate innervation in Alzheimer's disease. Neurosci Lett 1987;73:77- -80

50. Hyman BT, Van Hoesen GW, Damasio AR. Alzheimer's disease: glutamare depletion in the hippocampal perforant pathway zone. Ann Neurol 1987;22:37-40

51. Procter AW, Palmer AM, Francis PT, et al. Evidence of glutamatergic denervation and possible abnormal metabolism in $\mathrm{Alz}$ heimer's disease. J Neurochem 1988;50:790-802

52. Lowe SL, Bowen DM, Francis PT, Neary D. Ante mortem cerebral amino acid concentrations indicate selecrive degeneration of glutamate-enriched neurons in Alzheimer's disease. Neuroscience $1990 ; 38: 571-577$

53. Jagust WJ, Eberling JL, Richardson BC, et al. The cortical topography of temporal lobe hypometabolism in early Alzheimer's disease. Brain Res 1993;629:189-198

54. Foster NL, Minoshima S, Kuhl DE, Sima AAF. Metabolic topography of progressive limbic lobe sclerosis. J Cereb Blood Flow Metab 1995;15:\$818

55. Masliah E, Terry RD, Alford M, et al. Cortical and subcortical patterns of synaptophysinlike immunoreactivity in Alzheimer's disease. Am J Pathol 1991;138:235-246

56. Scheff SW, Sparks L, Price DA. Quantitative assessment of synaptic density in the entorhinal cortex in Alzheimer's disease. Ann Neurol 1993:34:356-361

57. Lynch G, Matchews DA, Mosko S, et al. Induced acetylcholinesterase-rich layer in rat dentate gyrus following entorhinal lesions. Brain Res 1972;42:311-318

58. Bertoni-Freddari C, Fattoretti P, Casoli T, et al. Morphological adaptive response of the synaptic junctional zones in the human dentate gyrus during aging and Alzheimer's disease. Brain Res 1990;517:69-75

59. Geddes JW, Monaghan DT, Cotman CW, et al. Plasticity of hippocampal circuitry in Alzheimer's disease. Science 1985;230: 1179-1181

60. Hyman BT, Kromer LJ, Van Hoesen GW. Reinnervation of the hippocampal perforant pathway zone in Alzheimer's disease. Ann Neurol 1987;21:259-267

61. Sutherland RJ, Whishaw IQ, Kolb B. Contributions of cingulate cortex to two forms of spatial learning and memory. J Neurosci 1988;8:1863-1872

62. Meunier M, Destrade C. Electrolytic but not ibotenic acid lesions of the posterior cingulate cortex produce transitory facilitation of learning in mice. Behav Brain Res 1988;27:161-172

63. Sif J, Meunier M, Messier C, et al. Quantitative $\left[{ }^{14} \mathrm{C}\right] 2$ deoxyglucose study of a functional dissociation between anterior and posterior cingulate cortices in mice. Neurosci Lett 1989; 101:223-228

64. Vogt BA, Finch DM, Olson CR. Functional heterogeneity in 
cingulate cortex: the anterior executive and posterior evaluative regions. Cereb Cortex 1992;2:435-443

65. Yukie M. Neural connections of auditory association cortex with the posterior cingulate cortex in the monkey. Neurosci Res 1995;22:179-187

66. Valenstein E, Bowers D, Verfaellic M, ct al. Retrosplenial amnesia. Brain 1987;110:1631-1646

67. Matsunami K, Kawashima T, Satake H. Mode of $\left[{ }^{14} \mathrm{C}\right]$ 2-deoxy-D-glucose uptake into retrosplenial cortex and other memory-related structures of the monkey during a delayed response. Brain Res Bull 1989;22:829-838

68. Neave N, Lloyd S, Sahgal A, Aggleton JP. Lack of effect of lesions in the anterior cingulate cortex and retrosplenial cortex on certain tests of spatial memory in the rat. Behav Brain Res 1994:65:89-101

69. Burdette JH, Minoshima S, Vander Borght T, et al. Alzheimer disease: improved visual interpretation of PET images by using three-dimensional stereotaxic surface projections. Radiology 1996;198:837-843

70. Vander Borght TM, Minoshima S, Giordani B, et al. Is regional cerebral glucose metabolism different between Alzheimer's and Parkinson's disease dementia with similar dementia severity? J Cereb Blood Flow Metab 1995;15:S788 